\title{
Freqüência de Alterações Hepáticas em Pacientes com Esclerodermia
}

\section{Frequency of Hepatic Abnormalities in Patients with Scleroderma}

\author{
Ariene Paixão ${ }^{(1)}$, Raimundo Paraná((2), Mittermayer Santiago ${ }^{(3)}$
}

\begin{abstract}
RESUMO
Objetivo: avaliar a freqüência das doenças hepáticas em pacientes com esclerodermia e, secundariamente, estudar a freqüência de infecção pelos vírus $\mathrm{B}$ e $\mathrm{C}$ da hepatite nesses pacientes, assim como a freqüência de auto-anticorpos séricos. Material e métodos: estudaram-se pacientes com diagnóstico de esclerodermia, localizada ou sistêmica, acompanhados no Ambulatório de Reumatologia do Hospital Santa Izabel. Como grupo de comparação, foram estudados pacientes com diagnóstico de acne vulgar. Resultados: dos 65 pacientes com diagnóstico de esclerodermia incluídos nesse trabalho, $35 \%$ apresentaram a gama-glutamiltransferase (gama-GT) alterada, 30\% tiveram a fosfatase alcalina aumentada e $17,1 \%$, a alaninoaminotransferase (ALT) acima dos valores de referência. A ALT apresentou-se mais alterada nos pacientes do que nos controles. Apenas um indivíduo dos 41 testados apresentou positividade para o anticorpo antimitocôndria enquanto $19 \%$ tinham anticorpo antimúsculo liso, não se observando diferença estatística na positividade desses anticorpos entre os dois grupos. Um paciente apresentou o HBsAg positivo e outro foi positivo para o anticorpo anti-HCV. Nenhum paciente apresentou manifestações clínicas de doença hepática. Conclusões: no presente estudo, embora as alterações de enzimas hepáticas em pacientes com esclerodermia não tenham sido incomuns, não se observou nenhum caso com manifestações clínicas de doença hepática.
\end{abstract}

Palavras-chave: esclerodermia, esclerose sistêmica, hepatopatia.

\section{INTRODUÇÃO}

A esclerodermia pode ter uma forma "puramente cutânea", dita esclerodermia localizada, que se apresenta em três formas clínicas: linear, morféia e morféia difusa ${ }^{(1)}$ ou com comprometimento de diversos órgãos e sistemas na forma denominada de esclerose sistêmica (ES). Eventualmente, esses pacientes apresentam alterações hepáticas que variam de leve alteração do perfil enzimático até um quadro completo de cirrose e/ou falência hepática.

\begin{abstract}
Introduction: to determine the frequency of hepatic disease in patients with scleroderma and, secondarily, to investigate the frequency of hepatitis $B$ and $C$ virus infection and determine the frequency of autoantibodies in this disease. Material and methods: patients with scleroderma followed at Hospital Santa Izabel were included in the study and patients with acne vulgaris served as a comparison group. Results: considering the 65 scleroderma patients, 35\% had elevated gamma-glutamyltranspeptidase (gamma-GT), 30\% had elevated alkaline phosphatase and 17.1\% had alanine aminotransferase (ALT) higher than the reference range. Raised ALT levels were more common in the scleroderma patients than in the control group. Nineteen percent (19\%) of the patients tested positive for anti-smooth muscle antibodies and only one patient had anti-mitocondria antibodies. There was no statistical difference between the two groups regarding antibody testing. Anti-HCV antibodies were observed in one patient and HBsAg was detected in another scleroderma patient. There was no patient with clinically significant hepatic disease. Conclusions: the present study showed that clinical hepatic disease did not occur in our scleroderma patients, despite the relatively increased frequency of liver enzymes abnormalities.
\end{abstract}

Keywords: scleroderma, systemic sclerosis, liver disease.

Essas alterações podem ser decorrentes do uso de medicações para o tratamento da doença ou em virtude de uma associação com doenças primárias de fígado como a cirrose biliar primária $(\mathrm{CBP})$ ou a hepatite auto-imune (HAI). Levando em conta a origem imunológica dessas doenças, mecanismos etiopatogênicos comuns são sugeridos, ainda que não completamente esclarecidos. O objetivo principal do presente trabalho foi avaliar a freqüência de alterações hepáticas em pacientes com esclerodermia e, secundaria-

Recebido em 16/06/05. Aprovado, após revisão, em 16/07/07. Declaramos a inexistência de conflitos de interesse.

Trabalho realizado no Serviço de Reumatologia do Hospital Santa Isabel, Salvador, Bahia.

1. Mestre em Medicina pela Universidade Federal da Bahia (UFBA)

2. Professor adjunto da Faculdade de Medicina da UFBA.

3. Professor adjunto da Escola Bahiana de Medicina e Saúde Pública (EBMSP).

Endereço para correspondência: Mittermayer Santiago, Praça Almeida Couto, 500, CEP 40000-000, Salvador, BA, e-mail: mitter@svn.com.br 
mente, estudar a freqüência de infecção pelos vírus B e C da hepatite e a freqüência de auto-anticorpos nessa doença.

\section{CASUÍSTICA, MATERIAL E MÉTODOS}

\section{PACIENTES}

A população-alvo do estudo foi constituída de pacientes com diagnóstico de esclerodermia, localizada ou sistêmica, acompanhados no Ambulatório de Reumatologia do Hospital Santa Izabel, na cidade do Salvador (BA). Os pacientes foram classificados como portadores de esclerodermia com base nos critérios do Colégio Americano de Reumatologia $^{(2)}$. Como grupo de comparação, foram estudados pacientes com diagnóstico de acne vulgar advindos do Ambulatório de Dermatologia da mesma instituição. Todos os pacientes eram atendidos pelo Sistema Único de Saúde (SUS). O projeto foi aprovado pelo Comitê de Ética em Pesquisa do mesmo hospital e todos os pacientes assinaram o termo de consentimento livre e esclarecido antes de entrar no estudo.

\section{EXAMES COMPLEMENTARES}

Além da avaliação clínica, os pacientes foram submetidos a uma avaliação complementar que incluiu exames laboratoriais: perfil bioquímico hepático e testes sorológicos especiais, como a pesquisa do fator antinuclear (FAN) por imunofluorescência indireta (IFI) em células HEp-2 (INOVA Diagnostics, San Diego, CA), anticorpos antimitocôndria e antimúsculo liso (IFI), sorologia para vírus B (HBsAg) e C (anti-HCV) da hepatite (ELISA, Sanofi-Pasteur, França).

\section{ANÁLISE ESTATÍSTICA}

Os resultados foram analisados pelo programa do software SPSS (versão 9.0). A comparação de médias foi feita pelo teste $t$ de Student para amostras independentes. A análise de proporções foi realizada pelo teste do quiquadrado e teste exato de Fisher. Os resultados com probabilidade $(\mathrm{p})$ do erro tipo I igual a $5 \%(\mathrm{p}<0,05)$ foram considerados significantes.

\section{RESULTADOS}

Estudaram-se 65 pacientes com diagnóstico de esclerodermia, dos quais $30(46,2 \%)$ apresentavam a forma sistêmica difusa da doença e $19(29,2 \%)$, a forma sistêmica limitada (CREST: calcinose, Raynaud, esofagopatia, esclerodactilia e teleangiectasias). Dezesseis pacientes $(24,6 \%)$ apresenta- vam a forma apenas cutânea da esclerodermia, dos quais 13 indivíduos (20\%) tinham a forma morféia em placa e 3 $(4,6 \%)$, a esclerodermia linear. A duração média da doença foi de 61,92 \pm 71,2 meses, variando de 1 a 30 anos.

A maioria dos pacientes era do sexo feminino $(81,5 \%)$, com idade média de 40,23 $\pm 16,7$ anos (variação de 3 a 76 anos). Quanto à etnia, observou-se que 38,5\% foram classificados como da raça mulata, seguidos por $33,8 \%$ da raça negra e $27,7 \%$, branca. As principais manifestações clínicas apresentadas pelos 49 pacientes com a forma sistêmica da doença são apresentadas na tabela 1 .

TABELA 1

MANIFESTAÇÕES CLÍNICAS APRESENTADAS PELOS 49 PACIENTES COM ESCLEROSE SISTÊMICA

\begin{tabular}{ll}
\hline Descrição & $\mathbf{n}(\%)$ \\
\hline Artralgia & $47(95,9)$ \\
\hline Fenômeno de Raynaud & $43(87,7)$ \\
\hline Micronecrose de polpas digitais & $27(55,1)$ \\
\hline Xerostomia & $29(59,1)$ \\
Xeroftalmia & $22(44,8)$ \\
\hline Fibrose pulmonar & $19(38,7)$ \\
\hline Disfagia & $18(36,7)$ \\
\hline Dispnéia & $18(36,7)$ \\
\hline Obstipação & $12(24,4)$ \\
\hline
\end{tabular}

Quanto aos testes laboratoriais realizados nos pacientes com esclerodermia, a análise do perfil bioquímico do fígado mostrou que 14 dos 42 pacientes (35\%) apresentaram gama-glutamiltransferase (gama-GT) alterada, 12 dos 42 (30\%) tiveram fosfatase alcalina aumentada e 7 de 41 pacientes $(17,1 \%)$ tiveram alaninoaminotransferase (ALT) acima dos valores de referência. A pesquisa de FAN foi positiva em 42 dos 50 pacientes (84\%). Apenas um indivíduo $(2,4 \%)$ dos 41 testados apresentou positividade para o anticorpo antimitocôndria, enquanto 8 de 42 (19\%) tinham anticorpo antimúsculo liso. Sorologia para hepatite B e hepatite $\mathrm{C}$ foi realizada em 41 pacientes. O HBsAg foi positivo em um paciente $(2,6 \%)$ e o anti-HCV, em apenas um caso $(2,6 \%)$. Nenhum paciente apresentava manifestações clínicas de doença hepática.

Os 65 pacientes com diagnóstico de esclerodermia incluídos no estudo foram comparados com 56 controles quanto à freqüência de manifestações clínicas e laboratoriais. Nessa análise, notou-se que não houve diferença estatística, na proporção de aumento de fosfatase alcalina e 
alteração do tempo de protrombina entre casos e controles. Por outro lado, a ALT apresentou-se mais alterada nos pacientes com esclerodermia que nos controles, enquanto a gama-GT apresentou uma tendência a ser mais elevada nos pacientes com esclerodermia, mas não atingiu níveis de significância estatística $(\mathrm{p}=0,066)$ (Tabela 2).

TABELA 2

AlteraÇÃo das ENZIMAS HEPÁTICAS NOS PORTADORES DE ESCLERODERMIA COMPARANDO-SE COM OS CONTROLES

\begin{tabular}{lccc}
\hline $\begin{array}{l}\text { Exames laboratoriais } \\
\mathbf{n}=\text { controle/caso }\end{array}$ & \multicolumn{2}{c}{$\mathbf{n ( \% )}$} & $\mathbf{p}$ \\
\hline$\uparrow \mathrm{FA}^{*}, \mathrm{n}=49 / 40$ & $10(20,4)$ & $12(30,0)$ & $\mathrm{p}=0,297$ \\
$\uparrow \gamma \mathrm{GT}^{* *}, \mathrm{n}=50 / 40$ & $9(18,0)$ & $14(35,0)$ & $\mathrm{p}=0,066$ \\
$\uparrow \mathrm{ALT}^{* * *}, \mathrm{n}=49 / 41$ & 0 & $7(17,1)$ & $\mathrm{p}<0,01$ \\
\hline
\end{tabular}

*FA = fosfatase alcalina. Valores de referência: 25 a $100 \mathrm{U} / \mathrm{L} ; * * \gamma \mathrm{GT}=$ gama-glutamiltransferase. Valores de referência: 2 a $30 \mathrm{U} / \mathrm{l}$; *** ALT = alaninoaminotransferase. Valores de referência: 10 a $40 \mathrm{U} / \mathrm{l}$.

Já em relação aos auto-anticorpos, pôde-se observar uma positividade para o FAN muito maior entre os casos de esclerodermia que entre os controles $(\mathrm{p}<0,001)$. Entretanto, não se observou diferença estatística na positividade para os anticorpos antimitocôndria nem antimúsculo liso entre os dois grupos. Nenhum paciente do grupo de comparação apresentava sorologia positiva para hepatite.

Os pacientes com esclerodermia na época do estudo utilizavam pelo menos uma das seguintes medicações: D-penicilamina, colchicina, ciclofosfamida, diltiazem, nifedipina, captopril, antiinflamatórios não-hormonais e prednisona.

\section{DISCUSSÃO}

Doenças hepáticas não têm sido consideradas uma significante característica da ES, e maior ênfase tem sido sempre dada às manifestações da doença no pulmão, na pele, no esôfago, nos rins e no coração. Curiosamente, em uma série de ES a freqüência de alterações hepáticas foi maior nos controles que nos pacientes ${ }^{(3)}$.

Segundo Murray Lyon et al.(4), os primeiros relatos de uma associação dessa doença com alterações hepáticas datam de 1934 quando Milbradt descreveu a presença de hepatoesplenomegalia em esclerodermia, observação esta que foi posteriormente confirmada por outros autores, inclusive com calcificação difusa dessas vísceras. A primeira descrição de cirrose foi feita em 1945, como "cirrose biliar crônica"(5).
Dois dos casos de ES descritos por Bartholomew et al. ${ }^{(6)}$ tinham características de CBP, bem como outros cinco casos descritos por Reynolds et al. ${ }^{(7)}$. A partir daí, alguns autores passaram a chamar a associação entre ES e CBP como "síndrome de Reynolds".

Dessa forma, embora a freqüência de manifestações hepáticas na ES não seja definitivamente conhecida, baseando-se nos resultados do presente estudo, conclui-se que a presença de doença hepática clinicamente sintomática é raramente vista. Contudo, tomando como base os relatos de casos previamente publicados na literatura, as doenças hepáticas mais freqüentemente associadas a ela são a $\mathrm{CBP}^{(4-8)}$, a $\mathrm{HAI}^{(9,10)} \mathrm{e}$, menos freqüentemente, a hiperplasia nodular regenerativa $^{(11)}$ e a colangite esclerosante primária ${ }^{(12)}$. Em geral, tais doenças hepáticas estão mais associadas à forma sistêmica limitada (CREST).

Por outro lado, embora a freqüência de CBP em ES pareça ser rara, até cerca de $15 \%$ dos pacientes com CBP têm evidência de esclerodermia, fazendo pensar na possibilidade de que tal associação possa ser mais do que coincidência, uma vez que ambas as doenças têm uma base auto-imune $^{(13)}$. Isso tem sido corroborado por estudos demonstrando a presença de anticorpos anticentrômero em associação com anticorpos antimitocondriais ${ }^{(14)}$.

Na presente série, não observamos casos com manifestação clínica de doença hepática. Por outro lado, alguns pacientes apresentaram alterações de enzimas hepáticas, e a elevação de ALT foi mais comum nos pacientes com esclerodermia que no grupo de comparação. Como não se observou maior freqüência de anticorpos antimúsculo liso nem antimitocôndria nos pacientes com esclerodermia, especula-se que não se pode atribuir essas alterações laboratoriais à existência de uma doença auto-imune hepática (CBP ou HAI) subclínica associada, embora a conclusão definitiva só poderia ser tirada a partir de estudo histopatológico de fígado, que não foi realizado no presente trabalho. Adicionalmente, não se pode excluir a possibilidade de que as alterações de enzimas hepáticas vistas nos pacientes com esclerodermia possam ser decorrentes do uso de medicações com potencial hepatotóxico, tais como D-penicilamina, ciclofosfamida, diltiazem, captopril e antiinflamatórios hormonais e não-hormonais.

Em conclusão, doença hepática clinicamente significante é um fenômeno raro em pacientes com esclerodermia, embora alterações laboratoriais caracterizadas por elevação de enzimas hepáticas possam ser vistas em boa parte dos pacientes. 


\section{REFERÊNCIAS}

1. LeRoy EC, Black C, Fleischmajer R, et al.: Scleroderma (systemic sclerosis): classification, subsets and pathogenesis. J Rheumatol 15: 202-5, 1988.

2. Preliminary criteria for the classification of systemic sclerosis (scleroderma). Subcommittee for scleroderma criteria of the American Rheumatism Association Diagnostic and Therapeutic Criteria Committee. Arthritis Rheum 23(5): 581-90, 1980.

3. D'Angelo WA, Fries JF, Masi AT, Shulman LE: Pathologic observations in systemic sclerosis (scleroderma). A study of fifty-eight autopsy cases and fifty-eight matched controls. Am J Med 46(3): 428-40, 1969.

4. Murray Lyon IM, Thompson RP, Ansell ID, Williams R: Scleroderma and primary biliary cirrhosis. Br Med J 1: 258-9, 1970.

5. Goetz RH, Berne MB: The pathology of progressive sistemic sclerosis (generalized scleroderma): with special reference to changes in the viscera. Clin Proc 4: 337-92, 1945.

6. Bartholomew LG, Cain JC, Winkelmann RK, Baggenstoss AH: Chronic disease of the liver associated with systemic scleroderma. Amer J Dig Dis 9: 43-55, 1964.

7. Reynolds TB, Denison EK, Frankl HD, Lieberman FL, Peters RL: New syndrome. Combination of primary biliary cirrhosis, scleroderma and hereditary hemorrhagic teliangectasia. Gastroenterology 58: 290, 1970.

8. Gonzalez-Lopez MA, Drake M, Gonzalez-Vela MC, Armesto S, Llaca HF, Val-Bernal JF: Generalized morphea and primary biliary cirrhosis coexisting in a male patient. J Dermatol 33(10): 709-13, 2006.

9. Khalifa M, Ben Jazia E, Hachfi W, Sriha B, Bahri F, Letaief A: Autoimmune hepatitis and morphea: a rare association. Gastroenterol Clin Biol 30(6-7): 917-8, 2006.

10. Marie I, Levesque H, Tranvouez JL, et al.: Autoimmune hepatitis and systemic sclerosis: a new overlap syndrome? Rheumatology (Oxford) 40(1): 102-6, 2001.

11. Kaburaki J, Kuramochi S, Fujii T, et al.: Nodular regenerative hyperplasia of the liver in a patient with systemic sclerosis. Clin Rheumatol 15(6): 613-6, 1996.

12. Fraile G, Rodriguez-Garcia JL, Moreno A: Primary sclerosing cholangitis associated with systemic sclerosis. Postgrad Med J 67(784): 189-92, 1991.

13. Miller F, Lane B, Soterakis J, D'Angelo WA: Primary biliary cirrhosis and scleroderma. The possibility of a common pathogenetic mechanism. Arch Pathol Lab Med 103: 505-9, 1979.

14. Abraham S, Begum S, Isenberg D: Hepatic manifestations of autoimmune rheumatic diseases. Ann Rheum Dis 63(2): 123-9, 2004. 